\title{
Proteobacteria Contain Diverse flg22 Epitopes That Elicit Varying Immune Responses in Arabidopsis thaliana
}

\author{
Janis H. T. Cheng, Melissa Bredow, Jacqueline Monaghan, and George C. diCenzo ${ }^{\dagger}$ \\ Department of Biology, Queen's University, Kingston ON, K7L 3N6, Canada \\ Accepted 7 February 2021.
}

Bacterial flagellin protein is a potent microbe-associated molecular pattern. Immune responses are triggered by a 22-aminoacid epitope derived from flagellin, known as flg22, upon detection by the pattern recognition receptor FLAGELLIN-SENSING2 (FLS2) in multiple plant species. However, increasing evidence suggests that flg22 epitopes of several bacterial species are not universally immunogenic to plants. We investigated whether flg22 immunogenicity systematically differs between classes of the phylum Proteobacteria, using a dataset of 2,470 flg22 sequences. To predict which species encode highly immunogenic flg22 epitopes, we queried a custom motif $\left({ }^{11}[\mathrm{ST}] \mathrm{xx}[\mathrm{DN}][\mathrm{DN}]\right.$ $\mathrm{xAGxxI}^{21}$ ) in the flg22 sequences, followed by sequence conservation analysis and protein structural modeling. These data led us to hypothesize that most flg22 epitopes of the $\gamma$ - and $\beta$-Proteobacteria are highly immunogenic, whereas most flg22 epitopes of the $\alpha-, \delta$-, and $\varepsilon$-Proteobacteria are weakly to moderately immunogenic. To test this hypothesis, we generated synthetic peptides representative of the flg22 epitopes of each proteobacterial class, and we monitored their ability to elicit an immune response in Arabidopsis thaliana. The flg22 peptides of $\gamma$ - and $\beta$-Proteobacteria triggered strong oxidative bursts, whereas peptides from the $\varepsilon-, \delta$-, and $\alpha$-Proteobacteria triggered moderate, weak, or no response, respectively. These data suggest flg22 immunogenicity is not highly conserved across the phylum Proteobacteria. We postulate that sequence divergence of each taxonomic class was present prior to the

\section{J. H. T. Cheng and M. Bredow contributed equally to this work. \\ ${ }^{\dagger}$ Corresponding author: G. C. diCenzo; george.dicenzo@queensu.ca}

Funding: J. H. T. Cheng was supported by a Natural Sciences and Engineering Research Council of Canada (NSERC) Undergraduate Summer Research Award. M. Bredow was supported by NSERC through a Postdoctoral Fellowship. Research in the J. Monaghan laboratory is supported by NSERC Discovery and Accelerator Grants, the Canada Research Chairs Program, and Queen's University, as well as infrastructure support through the John R Evans Leaders Fund from the the Canadian Foundation for Innovation and the Ontario Ministry of Research and Innovation. Research in the G. C. diCenzo laboratory is supported by an NSERC Discovery Grant, as well as research and infrastructure support from Queen's University.

*The $\boldsymbol{e}$-Xtra logo stands for "electronic extra" and indicates that there are supplementary materials published online.

The author(s) declare no conflict of interest.

Copyright $\odot 2021$ The Author(s). This is an open access article distributed under the CC BY 4.0 International license. evolution of FLS2, and that the ligand specificity of $A$. thaliana FLS2 was driven by the flg22 epitopes of the $\gamma$ - and $\beta$ Proteobacteria, a monophyletic group containing many common phytopathogens.

Keywords: bacterial pathogenesis, flagellin, flg22, MAMPs, plant immunity, Proteobacteria

Plants live in microbe-rich environments and have adapted complex relationships with their microbiota. Mutualistic plant-microbe interactions have placed strong selective pressures on plants to accommodate microbial communities that enhance the uptake of nutrients and contribute to plant fitness. At the same time, pathogenic plant-microbe interactions have placed strong selective pressures on plants to evolve a robust immune system capable of sensing and responding to danger. Key to this sense-and-response system are integral membrane receptor complexes that bind microbial ligands ("molecular patterns") with high affinity and trigger phosphorylationmediated signal transduction cascades (Couto and Zipfel 2016). Although often referred to as "pathogen"-associated molecular patterns, some scholars prefer the term "microbe"-associated molecular patterns (MAMPs), because these features are shared by entire classes of microbes, including pathogens, commensals, and mutualists (Ausubel 2005). How plants differentiate friend from foe, despite all groups of microbes containing MAMPs, remains unclear but is likely to involve complex, overlapping mechanisms (Plett and Martin 2018). This discrimination may depend on concurrent or subsequent release of plant-derived signals such as cellular debris or phytocytokines that bind additional receptors and amplify immune signaling (Gust et al. 2017). Pathogens and nonpathogens may also directly suppress immune signaling to gain access to plant hosts, or encode divergent MAMPs that evade detection by plant receptors (Gourion et al. 2015; Plett and Martin 2018).

One of the most well-known MAMPs is the bacterial flagellin protein (Felix et al. 1999). In several plant species, orthologs of the FLAGELLIN-SENSING2 (FLS2) receptor detect flagellin via a 22-amino-acid epitope known as flg22 (Felix et al. 1999; Gómez-Gómez and Boller 2000; Nicaise et al. 2009). Epitope flg22 is thought to be a broadly conserved and potent elicitor of plant immunity, based predominantly on studies using flagellin peptides of the genus Pseudomonas (class $\gamma$-Proteobacteria) (Felix et al. 1999). However, there is increasing evidence that the immunogenicity of flg22 epitopes differs between taxonomically diverse bacteria and is not 
necessarily correlated with bacterial lifestyle. For example, flg22 epitopes encoded by the plant pathogen Xanthomonas campestris $(\gamma$-Proteobacteria) and the plant commensal Paraburkholderia phytofirmans ( $\beta$-Proteobacteria) elicit an immune response in Arabidopsis thaliana, whereas flg22 epitopes from the plant mutualist Sinorhizobium meliloti $(\alpha$-Proteobacteria) and the plant pathogens Agrobacterium tumefaciens ( $\alpha$-Proteobacteria) and Ralstonia solanacearum ( $\beta$-Proteobacteria) do not induce an immune response in Arabidopsis thaliana or tomato (Bauer et al. 2001; Felix et al. 1999; Pfund et al. 2004; Sun et al. 2006; Trdá et al. 2014). These observations raise the possibility that the immunogenic properties of flg22 are not broadly conserved and that bacterial lifestyle is not a major driver of flg22 evolution.

To investigate the evolution of flg22 and its immunogenic properties, we performed an in silico survey of proteobacterial flg22 epitopes to predict which are likely to be highly immunogenic to $A$. thaliana. To accomplish this, we collected the amino acid sequences of 2,470 flagellin proteins from a representative set of 1,414 Proteobacteria (366 $\alpha$-Proteobacteria, $234 \beta$-Proteobacteria, $674 \gamma$-Proteobacteria, $74 \delta$-Proteobacteria, and 66 \&-Proteobacteria), and extracted flg22 sequences based on multiple sequence alignments and the positions of known flg22 epitopes (Supplementary File S1; Supplementary Dataset S1). This process yielded 2,404 peptides, encompassing 1,059 distinct peptide sequences. As an initial prediction of which flg22 epitopes might be highly immunogenic to A. thaliana, we queried the sequences for the motif ${ }^{11}[\mathrm{ST}] \mathrm{xx}[\mathrm{DN}][\mathrm{DN}]$ $\mathrm{xAGxxI}^{21}$, which includes amino acids important for activation of the FLS2-BRASSINOSTEROID INSENSITIVE1-ASSOCIATED KINASE1 (BAK1) immune signaling complex (Felix et al. 1999; Sun et al. 2013; Wei et al. 2020). We allowed for Ser or Thr at residue 11 because these amino acids have similar properties, while the flexibility of Asp or Asn at residues 14 and 15 was based on their functional interchangeability at residue 14 in the flg22 epitope of $X$. campestris (Sun et al. 2006). The ${ }^{11}[\mathrm{ST}] \mathrm{xx}$ $[\mathrm{DN}][\mathrm{DN}] \mathrm{xAGxxI}^{21}$ motif was found in the flg22 epitopes of $>90 \%$ of $\gamma$ - and $\beta$-Proteobacteria species that encode flagellin. In contrast, this motif was identified in the flg 22 epitopes of only 60,15 , and $2 \%$ of flagellin-encoding $\delta$-, $\alpha$-, and $\varepsilon$-Proteobacteria, respectively (Fig. 1; Supplementary Table S1; Supplementary Figs. S1 and S2). This result suggested that highly immunogenic flg22 epitopes are likely to be unevenly distributed across the Proteobacteria phylum.

The above suggestion was further supported by analysis of flg22 amino acid sequence conservation both within and across taxonomic classes (Fig. 2), and by in silico modeling of the interactions between representative flg22 peptides of each proteobacterial class (herein defined as consisting of the most commonly occurring amino acid at each position [Fig. 2], and termed flg22- $\gamma$, flg22- $\beta$, flg22- $\alpha$, flg22- $\delta$, and flg22- $\varepsilon$ ), in complex with FLS2 or BAK1 (Fig. 3; Supplementary Fig. S3). Analysis of protein interactions was carried out using the crystal structure of the flg22-bound FLS2/BAK1 complex (Sun et al. 2013) and visualized using PyMOL Molecular Graphics System (version 2.0; Schrödinger, LLC). MutaBind2 (Zhang et al. 2020) was used to predict changes in flg22 peptide binding affinity to FLS2 or binding affinity of flg22-bound FLS2 to BAK1 to simulate the two-step complex formation (Chinchilla et al. 2007; Sun et al. 2013) (Supplementary Table S2). To increase the amount of sequence diversity analyzed in the binding affinity predictions, we included a second peptide for each of the $\alpha-, \delta$-, and $\varepsilon$-Proteobacteria, using the second most commonly occurring amino acid at all positions where the frequencies of the two amino acids were nearly equal. In all, flg22- $\gamma$ and flg22- $\beta$ differed at a total of four and three positions, respectively, from the canonical flg22 sequence of Pseudomonas aeruginosa (flg22-Pae), known to induce a strong oxidative burst in several plants, including A. thaliana, tomato, and grapevine (Felix et al. 1999; Trdá et al. 2014). Nevertheless, flg22- $\gamma$ and flg22- $\beta$ were predicted to bind the FLS2/BAK1 receptor complex with high affinity (Supplementary Table S2). In contrast, nine of the flg22- $\alpha$ residues differed from flg22-Pae, and a strong decrease in binding to FLS2 $\left(\Delta \Delta \mathrm{G}_{\text {bind }}=2.37 \mathrm{kcal} / \mathrm{mol}\right)$ and BAK1 $\left(\Delta \Delta \mathrm{G}_{\text {bind }}=1.79 \mathrm{kcal} / \mathrm{mol}\right)$ was predicted. Similarly, the second flg22- $\alpha$ peptide was predicted to be strongly compromised in its ability to associate with FLS2 (Supplementary Table S2), the

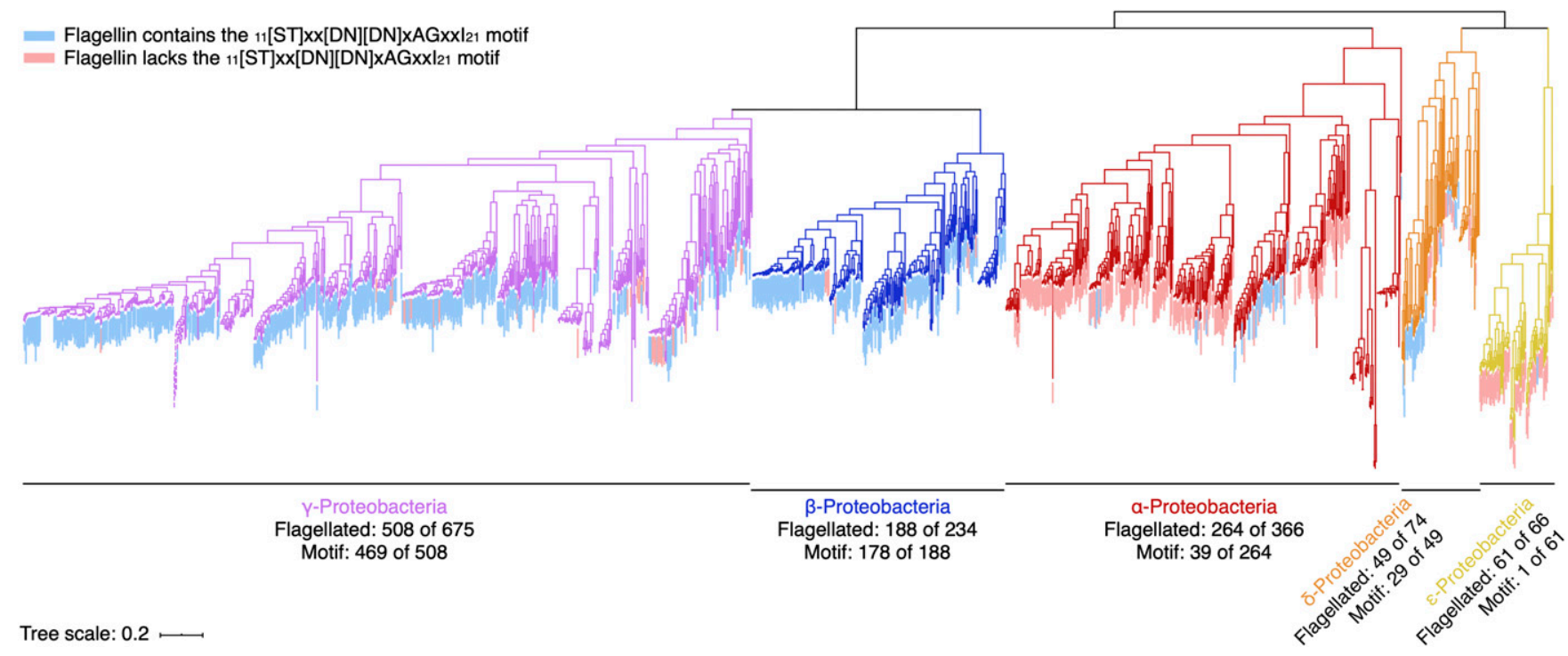

Fig. 1. Distribution of flagellin within the phylum Proteobacteria. A maximum-likelihood phylogeny of 1,414 Proteobacteria is shown, which was prepared from a concatenated alignment of 31 highly conserved proteins. Branches on the tree are color coded based on taxonomic class. Taxa encoding a flagellin containing the ${ }^{11}[\mathrm{ST}] \mathrm{xx}[\mathrm{DN}][\mathrm{DN}] \mathrm{xAGxxI}^{21}$ motif within flg22 are shown in blue; taxa only encoding flagellin that lack this motif are shown in pink, while taxa lacking flagellin are not colored. For each taxonomic class, the number of species containing flagellin is shown, as is the number of species containing the abovementioned motif. The scale bar represents the mean number of amino acid substitutions per site. Lengths of branches of a clade of $\gamma$-Proteobacteria endosymbionts (dashed lines) were reduced for presentation purposes. A larger version of this figure, with legible taxa names and the location of some known plant pathogens highlighted, is provided as Supplementary Figure S1. 
prerequisite first step of FLS2-flg22-BAK1 complex formation. Epitope flg22- $\delta$ differed from flg22-Pae at seven residues, although no impact on binding to FLS2 or BAK1 was predicted. However, formation of the FLS2-flg22-BAK1 complex could be negatively impacted by the Arg at position 22 , which is positively charged and larger than the Ser or Ala in flg22- $\gamma$ or flg22- $\beta$; because residue 22 is missing from the crystal structure (Sun et al. 2013), we were unable to test this hypothesis in silico. Epitope

\section{A $\gamma$-Proteobacteria - flg22- $\gamma$ - ERLSSGLRINSAKDDAAGLQIS}

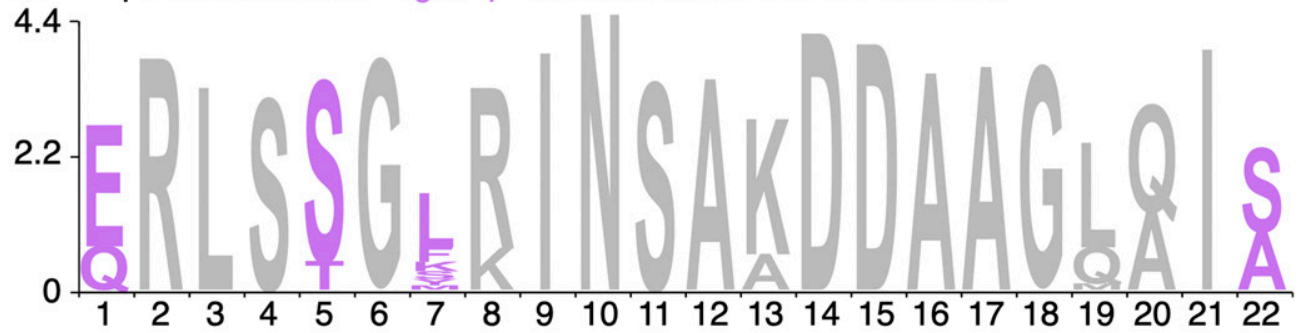

B

$\beta$-Proteobacteria - flg22- $\beta$ - QRLSSGLRINSAKDDAAGLAIA
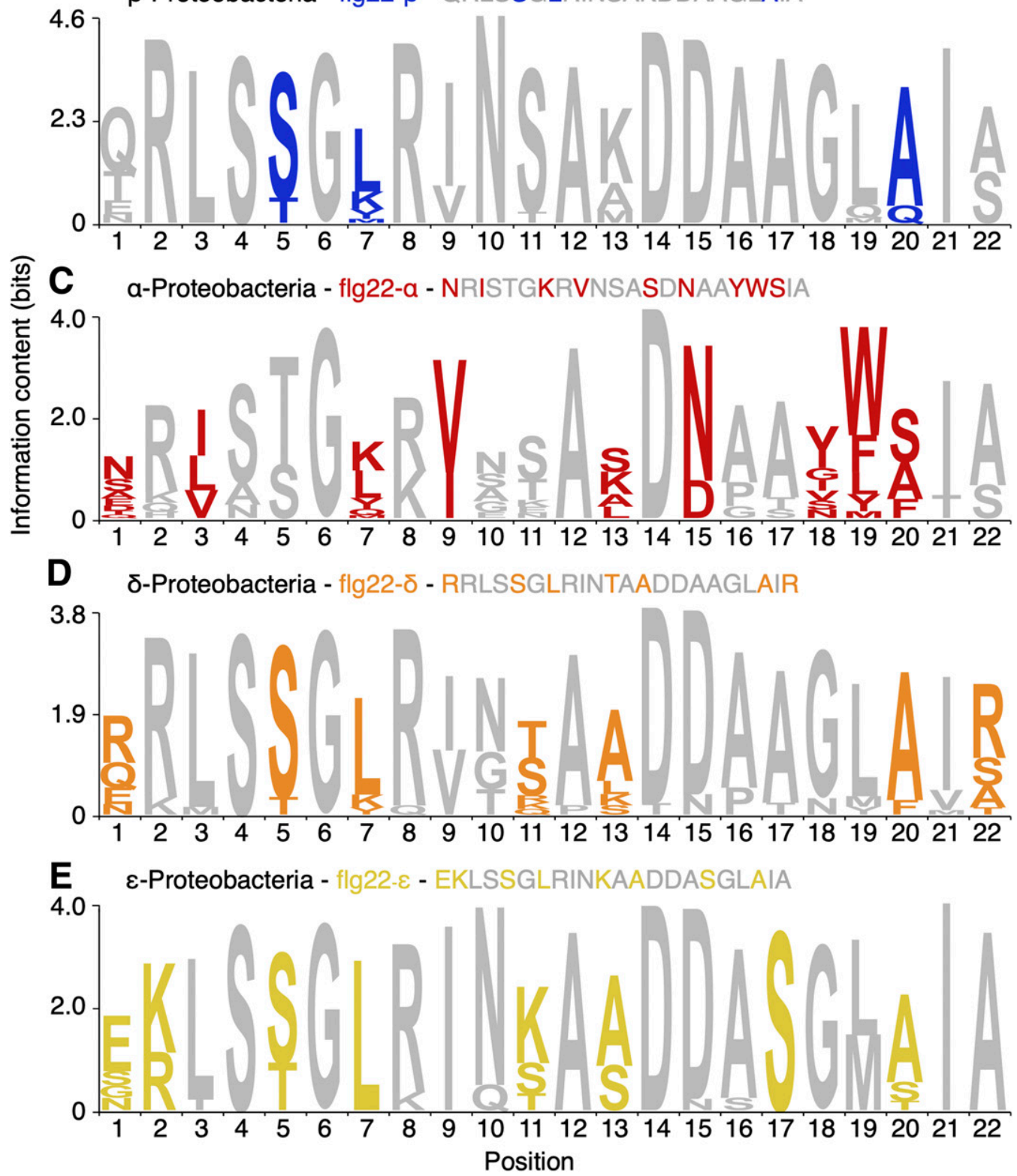

Fig. 2. Sequence conservation of the flg 22 epitopes of the phylum Proteobacteria. A to E, Amino acid logos of the flg 22 epitopes of five proteobacterial classes are shown. Logos were generated using Skylign (Wheeler et al. 2014) based on all of the flg22 epitopes identified in each taxonomic class. For each logo, colors indicate the positions that differ (defined as having a different most-common amino acid at that position) from the flg22 sequence of Pseudomonas aeruginosa. The representative sequence (defined as consisting of the most commonly occurring amino acid at each position) of each taxonomic class is given above each logo. For comparison, the canonical flg22 sequence of $P$. aeruginosa (flg22-Pae) is QRLSTGSRINSAKDDAAGLQIA. 

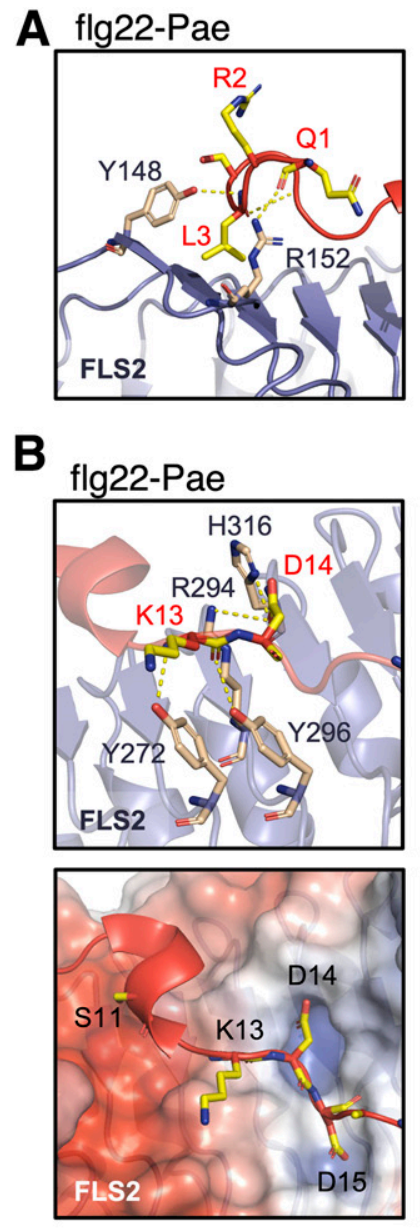

C flg22-Pae
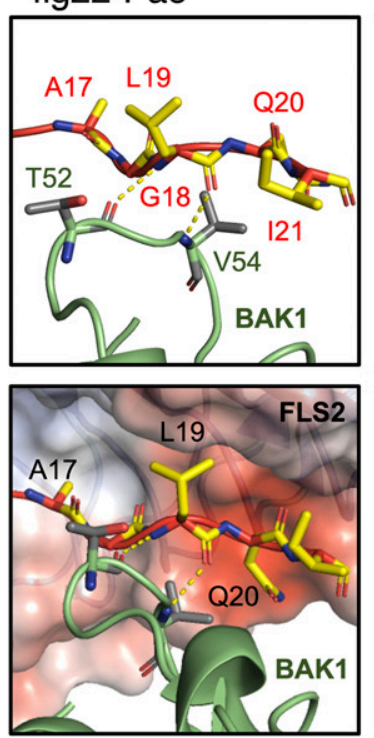

flg22- $\alpha$

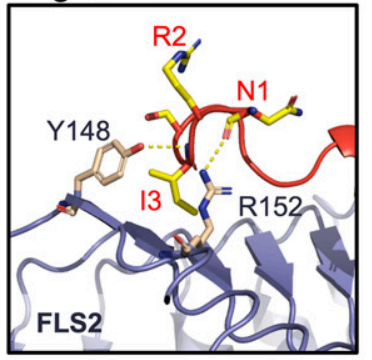

flg22- $\alpha$
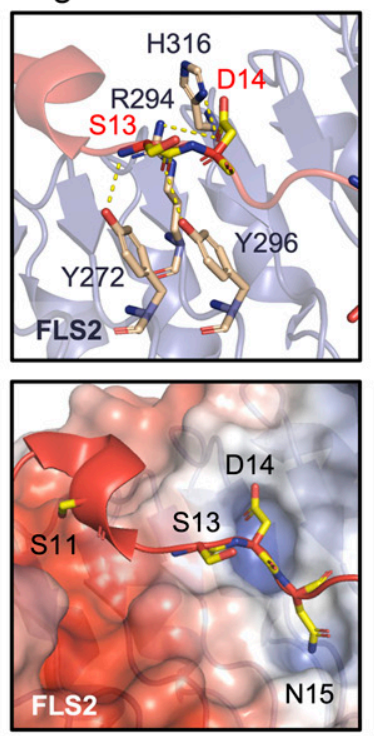

$f \lg 22-\alpha$
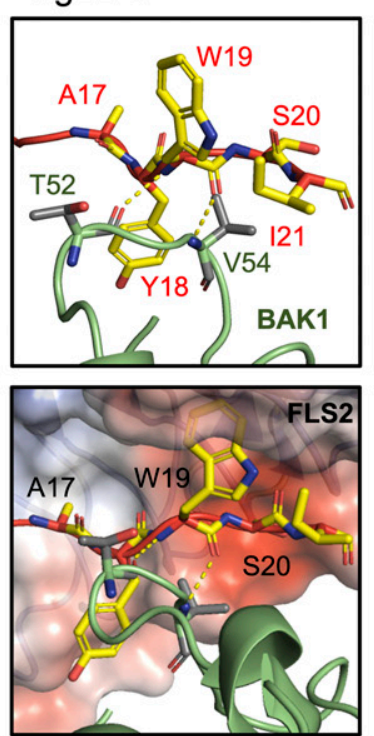

flg22- 6

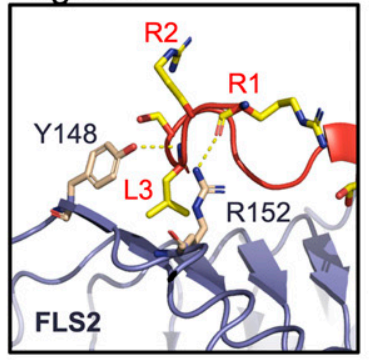

$\mathrm{flg} 22-6$
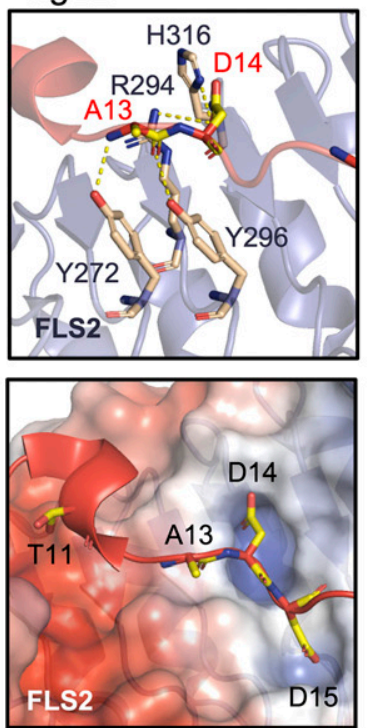

flg22- 6
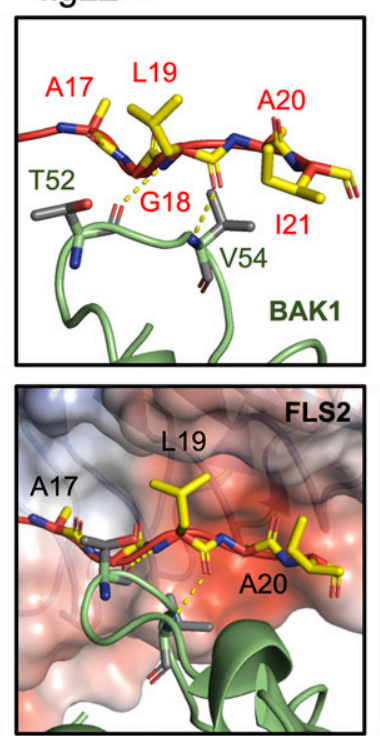

flg22- $\varepsilon$

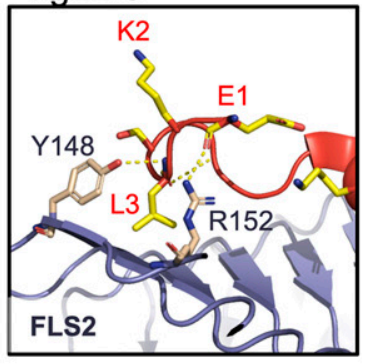

flg22- $\varepsilon$
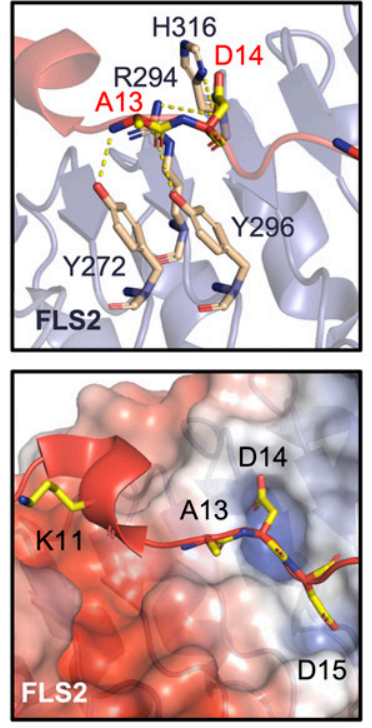

flg22- $\varepsilon$
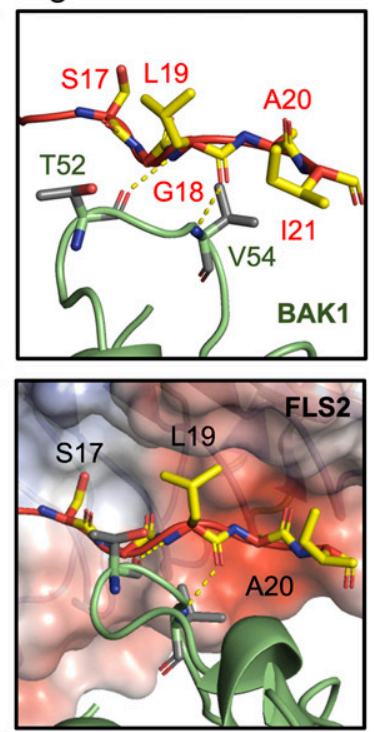

Fig. 3. Structural modeling of the FLS2-flg22-BAK1 complex using peptide sequences derived from $\alpha-, \delta$-, and $\varepsilon$-Proteobacteria. A, Association between the amino-terminal portion of flg22 (positions 1 to 9) (red) and FLS2 (purple). The flg22 epitope encoded by Pseudomonas aeruginosa (Pae) and FLS2 is stabilized by H-bonds (dotted yellow lines) between the backbone of flg22 at Gln-1 (Q1) and Leu-3 (L3) and the side chains of Tyr-148 (Y148) and Arg-152 (R152) of FLS2, respectively (Sun et al. 2013). H-bonding is predicted at these positions for all modeled peptides. B, Interaction between the central portion of flg22 (positions 10 to 15) and FLS2 is stabilized by H-bonds between Tyr-272 (Y272), Arg-294 (R294), Tyr-296 (Y296), and His-316 (H316) of FLS2 and Lys-13 (K13) and Asp-14 (D14) of flg22 (Sun et al. 2013), which is predicted for all flg22 peptides. Asp-14 (D14) and Asp-15 (D15) of flg22-Pae interact with two positively charged pockets on the surface of FLS2 (blue) (Sun et al. 2013). Substitution of Asp-15 with Asn-15 in flg22- $\alpha$ is not expected to impact interaction with FLS2 (Sun et al. 2006). C, The carboxyl-terminal portion of flg22 (positions 16 to 22) interacts with FLS2 and BAK1 (green). Leu-19 (L19) of flg22 forms H-bonds with Thr-52 (T52) and Val-54 (V54) of BAK1 (Sun et al. 2013) and interacts with a hydrophobic pocket (red) on the surface of FLS2 (Sun et al. 2013). Tyr and Trp residues at positions 18 (Y18) and 19 (W19) of flg22- $\alpha$, respectively, are predicted to cause steric hindrance between FLS2 and BAK1. The Ser residue at position 17 (S17) of flg22- $\varepsilon$ may cause slight steric hindrance with FLS2. 

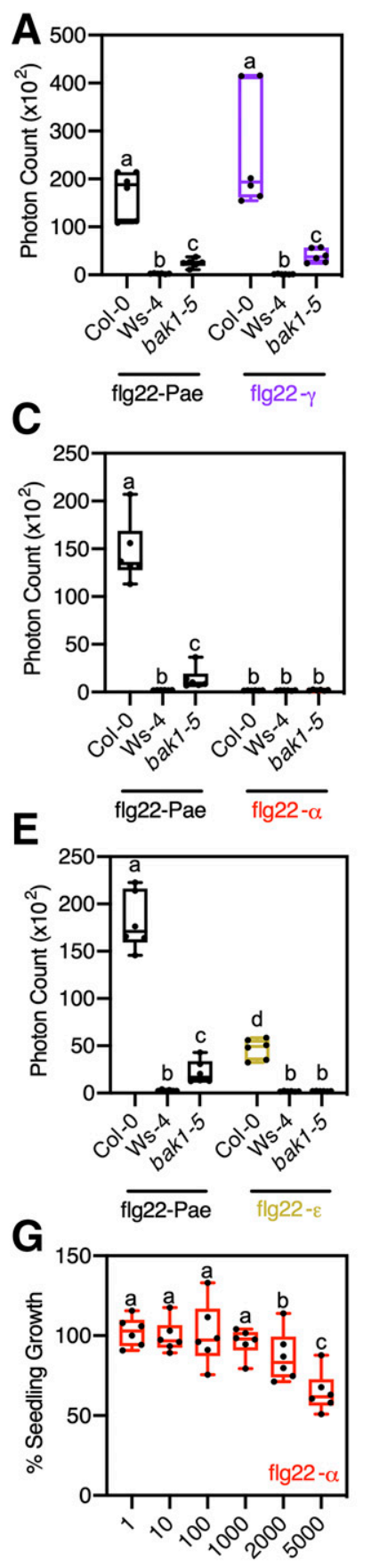

Concentration (nM)
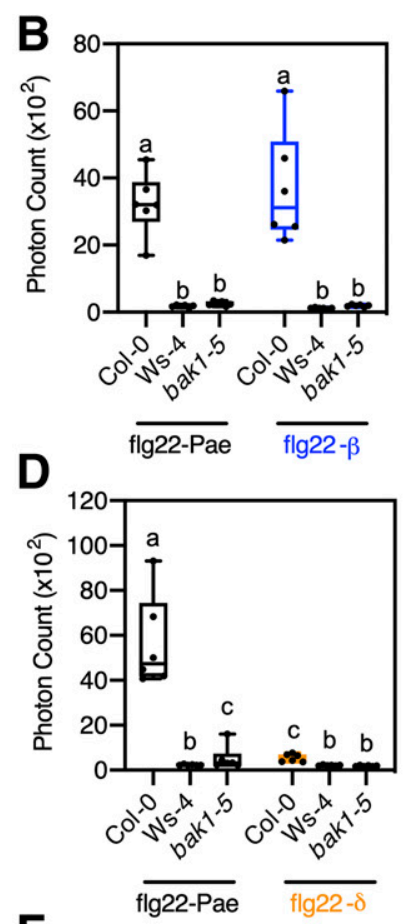

$F$
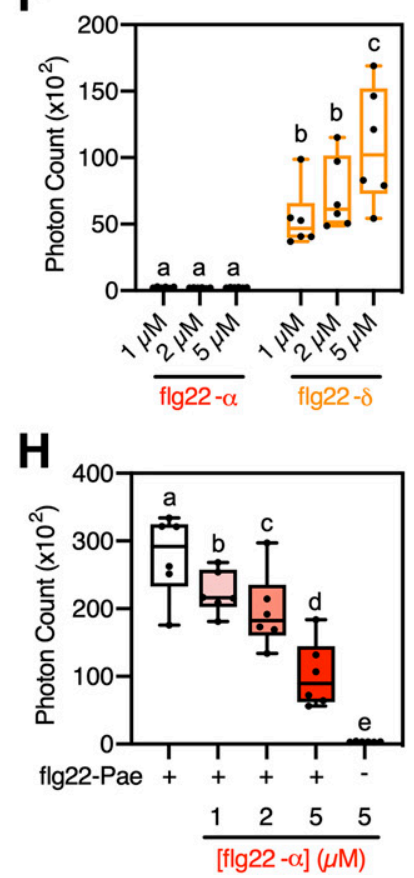

Fig. 4. Immunogenicity of proteobacterial flg 22 peptides. A to E, Oxidative species production in Col-0, bak1-5, and Ws-4 leaf discs following treatment with 100 nM A, flg22- $\gamma$; B, flg22- $\beta$; C, flg22- $\alpha$; D, flg22- $\delta$; or E, flg22- $\varepsilon$ peptides. Results for the flg22-Pae peptide are shown in each panel for comparison. Cumulative photon count was measured over $40 \mathrm{mins}$. F, The flg22- $\alpha$ and flg22- $\delta$-elicited oxidative bursts in Col-0 leaf discs using 1 to $5 \mu \mathrm{M}$ each peptide. $\mathbf{G}$, Seedling growth inhibition of Col-0 seedlings following continual treatment with 1 to $5,000 \mathrm{nM}$ flg22- $\alpha$ for 14 days. Values are normalized to Col-0 seedlings grown in the absence of flg 22 . Data for the other flg22 peptides are shown in Supplementary Figure S5. H, Oxidative species production in Col-0 leaf discs following elicitation with $100 \mathrm{nM}$ flg22-Pae and 1 to $5 \mu \mathrm{M}$ flg22- $\alpha$. Data are presented as boxplots indicating first and third quartiles, split by a median line. Whiskers represent maximum and minimum values. Each experiment consisted of six replicates and was repeated three independent times with similar results; results for one representative experiment are shown. Statistically different $(P<0.05)$ values are denoted by different lowercase letters according to a one-way analysis of variance followed by a Tukey's posthoc test using GraphPad Prism8. flg22- $\varepsilon$ differed from flg22-Pae at eight residues. Binding of flg22$\varepsilon$ to FLS2 was predicted to be moderately decreased $\left(\Delta \Delta \mathrm{G}_{\text {bind }}=\right.$ $0.81 \mathrm{kcal} / \mathrm{mol}$ ) compared with flg22-Pae, with protein structural modeling suggesting that this may be partially due to slight steric hindrance caused by the Ser-17 in flg22- $\varepsilon$ (Fig. 3C). No significant differences between the binding affinity for the first and second versions of the flg22- $\delta$ and flg22- $\varepsilon$ peptides were predicted (Supplementary Table S2).

Together, the motif prevalence, sequence analysis, and protein modeling data led us to hypothesize that most flg22 epitopes of the $\gamma$ - and $\beta$-Proteobacteria are highly immunogenic, whereas most flg22 epitopes of the $\alpha-, \delta$-, and $\varepsilon$-Proteobacteria are weakly to moderately immunogenic. To test this hypothesis, we generated synthetic peptides of the five representative flg22 sequences (Fig. 2), and monitored their ability to elicit an oxidative burst in A. thaliana leaf tissue. Assays using leaf disks from the bak1-5 mutant (Schwessinger et al. 2011) and accession Ws-4 (a natural fls 2 null mutant) (Gómez-Gómez and Boller 2000) confirmed that the oxidative bursts observed in Col-0 are FLS2/BAK1 dependent (Fig. 4A to E). Strikingly, and consistent with our hypothesis, only flg $22-\gamma$ or flg22- $\beta$ generated oxidative bursts comparable with flg22-Pae in A. thaliana Col-0 plants when applied at a concentration of $100 \mathrm{nM}$ (Fig. $4 \mathrm{~A}$ and B; Supplementary Fig. S4). In contrast, the oxidative bursts of $A$. thaliana Col-0 leaves exposed to $100 \mathrm{nM}$ flg22- $\varepsilon$ and flg $22-\delta$ were approximately 70 and $90 \%$ lower, respectively, than those induced by $100 \mathrm{nM}$ flg22-Pae, while no detectable oxidative burst was induced using $100 \mathrm{nM}$ flg22- $\alpha$ (Fig. 4C to E; Supplementary Fig. S4). Increasing the concentration of flg22- $\delta$ led to dose-dependent oxidative bursts, confirming that flg22- $\delta$ is weakly immunogenic to A. thaliana (Fig. $4 \mathrm{~F})$. On the other hand, no detectable oxidative burst was induced by flg22- $\alpha$ even at $5 \mu \mathrm{M}$ (Fig. 4F). However, in seedling growth inhibition assays (Fig. 4G; Supplementary Fig. S5), 2 and $5 \mu \mathrm{M}$ flg22- $\alpha$ did result in approximately 20 and $40 \%$ reductions in seedling weight, respectively, suggesting that this peptide may weakly associate with the FLS2/BAK1 complex and eventually trigger a weak immune response in A. thaliana following prolonged exposure. In support of this, a dose-dependent decrease in $100 \mathrm{nM}$ flg22-Pae-induced oxidative bursts was observed when simultaneously applied to A. thaliana Col-0 leaves with 1,2 , or $5 \mu \mathrm{M}$ flg22- $\alpha$ as a competitor (Fig. $4 \mathrm{H}$ ). This result mirrors those observed with the flg22- $\Delta \mathrm{A}^{16 / 17}$ peptide (Chinchilla et al. 2006), and suggests that flg22- $\alpha$ is a competitive antagonist able to weakly bind the FLS2/BAK1 complex while activating little to no downstream signaling.

The observations made here (Fig. 4H) and elsewhere (Chinchilla et al. 2006) that nonimmunogenic flg22 peptides can compete for FLS2 binding raise the exciting possibility that community dynamics within a plant's microbiota, whose composition differs across tissues (Turner et al. 2013) and environments (Dastogeer et al. 2020), may modulate FLS2-dependent immune responses. In particular, FLS2-dependent immune responses may be locally suppressed by high densities of microbes with nonimmunogenic flg22 epitopes that remain capable of interacting with FLS2/BAK1, while plants or tissues lacking these microbes may display more sensitive FLS2-dependent pathogen detection. However, it is important to keep in mind that the mere presence or absence of immunogenic flg22 epitopes does not indicate whether or not a microbe is phytopathogenic. For example, in the $\beta$-Proteobacteria, Ralstonia phytopathogens carry flg22 epitopes that are nonimmunogenic in A. thaliana and tomato (Pfund et al. 2004; Mueller et al. 2012), whereas the plant mutualists of the sister genus Cupriavidus carry flg22 epitopes that are likely to be immunogenic (Fig. 1; Supplementary Fig. S1). Similarly, in the $\alpha$-Proteobacteria, Agrobacterium phytopathogens carry flg22 epitopes that are 
nonimmunogenic to Arabidopsis (Felix et al. 1999), whereas species in the order Sphingomonadales, commonly found in plant leaf microbial communities (Delmotte et al. 2009; Chaudhry et al. 2021), carry flg22 epitopes that we predict to be highly immunogenic (Fig. 1; Supplementary Fig. S1). Thus, any potential impact of community composition on the sensitivity of FLS2-dependent pathogen detection cannot simply be explained by competition between beneficial versus pathogenic microbes.

Our data clearly demonstrate that the immunogenicity of flg22 epitopes varies across the phylum Proteobacteria, with highly immunogenic flg22 epitopes predominantly found in the $\gamma$ and $\beta$-Proteobacteria. We postulate that this observation relates to the timing of FLS2 evolution relative to the divergence of the proteobacterial classes. The $\alpha$-Proteobacteria are estimated to have diverged from the $\gamma$ - and $\beta$-Proteobacteria between 2,000 and 3,000 million years ago (mya) (Battistuzzi et al. 2004). In contrast, FLS2 likely emerged following the evolution of land plants (approximately 500 mya) and prior to the diversification of the Spermatophyta (approximately 350 mya) (Albert et al. 2010; de Vries et al. 2018; Morris et al. 2018). We suggest that the divergence of flg22 sequences between proteobacterial classes was not driven by selection to evade plant immune detection, although this may be important in explaining flg22 sequence divergence at finer taxonomic scales. Instead, we hypothesize that the flg22 sequences of the proteobacterial classes were already diverged prior to the emergence of FLS2, and that evolution of FLS2 ligand specificity was driven by the flg 22 epitopes of the $\gamma$ - and $\beta$-Proteobacteria, a monophyletic group containing many common phytopathogens.

If the above hypothesis is true, it raises the interesting question of why most plant pathogens have not evolved nonimmunogenic versions of flg22. We speculate this could be due to several, nonmutually exclusive reasons. The term "pathogen" is context dependent and most pathogenic microbes also have nonpathogenic lifestyles; for a nice discussion of this topic, see Casadevall and Pirofski (2014). It is possible that substitutions within the flg22 region of flagellin result in fitness trade-offs such as reduced motility that are selected against. In addition, phytopathogens often encode effectors that can block FLS2-mediated immune responses (Shan et al. 2008), which could reduce the evolutionary advantage of losing flg22 immunogenicity. Finally, some phytopathogens do carry noncanonical flg22 epitopes as discussed above (Fig. 1; Supplementary Fig. S1) and, similarly, there can be variation in FLS2 across even closely related plant species (Vetter et al. 2012). Notably, recent evidence suggests that the evolution of noncanonical flg22 epitopes by phytopathogens can be countered by coevolution in FLS2. For example, the FLS2 protein of Glycine max (soybean) can trigger an immune response upon exposure to the flg22 epitope of $R$. solanacearum, whereas this flg22 epitope is nonimmunogenic to A. thaliana (Wei et al. 2020). Similarly, the flg22 epitope of the phytopathogen Agrobacterium tumefaciens is a classic example of a "nonimmunogenic" flg22 epitope based on studies with A. thaliana and tomato (Felix et al. 1999); however, it is recognized by the FLS2 ${ }^{\mathrm{XL}}$ protein of Vitis riparia (riverbank grape), leading to a strong immune response (Fürst et al. 2020). Thus, it is possible that the advantages of a phytopathogen carrying a noncanonical flg22 epitope may be masked by negative trade-offs, and that any advantages are short lived due to the potential for coevolution of FLS2.

\section{Data availability.}

Scripts to repeat the computational analyses reported in this study are available online at GitHub.

\section{ACKNOWLEDGMENTS}

This research was enabled, in part, through computational resources provided by Compute Ontario and Compute Canada.

\section{AUTHOR-RECOMMENDED INTERNET RESOURCES}

Compute Canada: https://www.computecanada.ca Compute Ontario: https://www.computeontario.ca GitHub: https://github.com/diCenzo-GC/Proteobacterial_flg22

\section{LITERATURE CITED}

Albert, M., Jehle, A. K., Lipschis, M., Mueller, K., Zeng, Y., and Felix, G. 2010. Regulation of cell behaviour by plant receptor kinases: Pattern recognition receptors as prototypical models. Eur. J. Cell Biol. 89: 200-207.

Ausubel, F. M. 2005. Are innate immune signaling pathways in plants and animals conserved? Nat. Immunol. 6:973-979.

Battistuzzi, F. U., Feijao, A., and Hedges, S. B. 2004. A genomic timescale of prokaryote evolution: Insights into the origin of methanogenesis, phototrophy, and the colonization of land. BMC Evol. Biol. 4:44.

Bauer, Z., Gómez-Gómez, L., Boller, T., and Felix, G. 2001. Sensitivity of different ecotypes and mutants of Arabidopsis thaliana toward the bacterial elicitor flagellin correlates with the presence of receptorbinding sites. J. Biol. Chem. 276:45669-45676.

Casadevall, A., and Pirofski, L. A. 2014. Microbiology: Ditch the term pathogen. Nature 516:165-166.

Chaudhry, V., Runge, P., Sengupta, P., Doehlemann, G., Parker, J. E., and Kemen, E. 2021. Shaping the leaf microbiota: Plant-microbe-microbe interactions. J. Exp. Bot. 72:36-56.

Chinchilla, D., Bauer, Z., Regenass, M., Boller, T., and Felix, G. 2006. The Arabidopsis receptor kinase FLS2 binds flg22 and determines the specificity of flagellin perception. Plant Cell 18:465-476.

Chinchilla, D., Zipfel, C., Robatzek, S., Kemmerling, B., Nürnberger, T., Jones, J. D. G., Felix, G., and Boller, T. 2007. A flagellin-induced complex of the receptor FLS2 and BAK1 initiates plant defence. Nature 448:497-500.

Couto, D., and Zipfel, C. 2016. Regulation of pattern recognition receptor signalling in plants. Nat. Rev. Immunol. 16:537-552.

Dastogeer, K. M. G., Tumpa, F. H., Sultana, A., Akter, M. A., and Chakraborty, A. 2020. Plant microbiome-An account of the factors that shape community composition and diversity. Curr. Plant Biol. 23: 100161.

Delmotte, N., Knief, C., Chaffron, S., Innerebner, G., Roschitzki, B., Schlapbach, R., von Mering, C., and Vorholt, J. A. 2009. Community proteogenomics reveals insights into the physiology of phyllosphere bacteria. Proc. Natl. Acad. Sci. U.S.A. 106:16428-16433.

de Vries, S., de Vries, J., von Dahlen, J. K., Gould, S. B., Archibald, J. M., Rose, L. E., and Slamovits, C. H. 2018. On plant defense signaling networks and early land plant evolution. Commun. Integr. Biol. 11:1-14.

Felix, G., Duran, J. D., Volko, S., and Boller, T. 1999. Plants have a sensitive perception system for the most conserved domain of bacterial flagellin. Plant J. 18:265-276.

Fürst, U., Zeng, Y., Albert, M., Witte, A. K., Fliegmann, J., and Felix, G 2020. Perception of Agrobacterium tumefaciens flagellin by FLS2 $2^{\mathrm{XL}}$ confers resistance to crown gall disease. Nat. Plants 6:22-27.

Gómez-Gómez, L., and Boller, T. 2000. FLS2: An LRR receptor-like kinase involved in the perception of the bacterial elicitor flagellin in Arabidopsis. Mol. Cell 5:1003-1011.

Gourion, B., Berrabah, F., Ratet, P., and Stacey, G. 2015. Rhizobiumlegume symbioses: The crucial role of plant immunity. Trends Plant Sci. 20:186-194.

Gust, A. A., Pruitt, R., and Nürnberger, T. 2017. Sensing danger: Key to activating plant immunity. Trends Plant Sci. 22:779-791.

Morris, J. L., Puttick, M. N., Clark, J. W., Edwards, D., Kenrick, P., Pressel, S., Wellman, C. H., Yang, Z., Schneider, H., and Donoghue, P. C. J. 2018 The timescale of early land plant evolution. Proc. Natl. Acad. Sci. U.S.A. 115:E2274-E2283.

Mueller, K., Bittel, P., Chinchilla, D., Jehle, A. K., Albert, M., Boller, T., and Felix, G. 2012. Chimeric FLS2 receptors reveal the basis for differential flagellin perception in Arabidopsis and tomato. Plant Cell 24:2213-2224.

Nicaise, V., Roux, M., and Zipfel, C. 2009. Recent advances in PAMPtriggered immunity against bacteria: Pattern recognition receptors watch over and raise the alarm. Plant Physiol. 150:1638-1647. 
Pfund, C., Tans-Kersten, J., Dunning, F. M., Alonso, J. M., Ecker, J. R., Allen, C., and Bent, A. F. 2004. Flagellin is not a major defense elicitor in Ralstonia solanacearum cells or extracts applied to Arabidopsis thaliana. Mol. Plant-Microbe Interact. 17:696-706.

Plett, J. M., and Martin, F. M. 2018. Know your enemy, embrace your friend: Using omics to understand how plants respond differently to pathogenic and mutualistic microorganisms. Plant J. 93:729-746.

Schwessinger, B., Roux, M., Kadota, Y., Ntoukakis, V., Sklenar, J., Jones, A., and Zipfel, C. 2011. Phosphorylation-dependent differential regulation of plant growth, cell death, and innate immunity by the regulatory receptor-like kinase BAK1. PLoS Genet. 7:e1002046.

Shan, L., He, P., Li, J., Heese, A., Peck, S. C., Nürnberger, T., Martin, G. B., and Sheen, J. 2008. Bacterial effectors target the common signaling partner BAK1 to disrupt multiple MAMP receptor-signaling complexes and impede plant immunity. Cell Host Microbe 4:17-27.

Sun, W., Dunning, F. M., Pfund, C., Weingarten, R., and Bent, A. F. 2006. Within-species flagellin polymorphism in Xanthomonas campestris pv campestris and its impact on elicitation of Arabidopsis FLAGELLIN SENSING2-dependent defenses. Plant Cell 18:764-779.

Sun, Y., Li, L., Macho, A. P., Han, Z., Hu, Z., Zipfel, C., Zhou, J.-M., and Chai, J. 2013. Structural basis for flg22-induced activation of the Arabidopsis FLS2-BAK1 immune complex. Science 342:624628 .
Trdá, L., Fernandez, O., Boutrot, F., Héloir, M.-C., Kelloniemi, J., Daire, X., Adrian, M., Clément, C., Zipfel, C., Dorey, S., and Poinssot, B. 2014. The grapevine flagellin receptor VvFLS2 differentially recognizes flagellin-derived epitopes from the endophytic growth-promoting bacterium Burkholderia phytofirmans and plant pathogenic bacteria. New Phytol. 201:1371-1384.

Turner, T. R., James, E. K., and Poole, P. S. 2013. The plant microbiome. Genome Biol. 14:209.

Vetter, M. M., Kronholm, I., He, F., Häweker, H., Reymond, M., Bergelson, J., Robatzek, S., and de Meaux, J. 2012. Flagellin perception varies quantitatively in Arabidopsis thaliana and its relatives. Mol. Biol. Evol. 29:1655-1667.

Wei, Y., Balaceanu, A., Rufian, J. S., Segonzac, C., Zhao, A., Morcillo, R. J. L., and Macho, A. P. 2020. An immune receptor complex evolved in soybean to perceive a polymorphic bacterial flagellin. Nat. Commun. 11: 3763.

Wheeler, T. J., Clements, J., and Finn, R. D. 2014. Skylign: A tool for creating informative, interactive logos representing sequence alignments and profile hidden Markov models. BMC Bioinf. 15:7.

Zhang, N., Chen, Y., Lu, H., Zhao, F., Alvarez, R. V., Goncearenco, A., Panchenko, A. R., and Li, M. 2020. MutaBind2: Predicting the impacts of single and multiple mutations on protein-protein interactions. iScience 23:100939. 